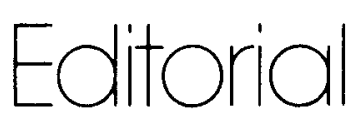

\title{
Minimizing Antimicrobial Resistance in Hospital Bacteria: Can Switching or Cycling Drugs Help?
}

\author{
John E. McGowan, Jr., MD
}

During the past decade, some pathogens in the hospi.tal, and in the community as well, have developed resistance to the antimicrobial drugs in current use. ${ }^{1}$ Use of antimicrobial agents is one of the factors leading to resis.tance in microorganisms. ${ }^{2,3}$ Existence of this cause and effect relationship forces us to examine the ways in which we use antibiotics.

It has been postulated that switching to a new drug of the same family can reduce levels of resistance to older drugs, and that "cycling" antimicrobials (periodically substituting a new member of the same drug family for a Fdrug currently in use) might minimize development of resistance to specific drugs in the same family. ${ }^{4} \mathrm{~A}$ number of recent studies ${ }^{5-19}$ have attempted to discern the effectiveness of these strategies (Table). The majority of these trials have substituted amikacin for gentamicin or tobramycin in hopes of reducing prevalence of resistance to drugs of this group. This review addresses two major questions about these practices: Do they work? and Are they cost-effective?

\footnotetext{
From the Department of Pathology and Laboratory Medicine, Emory University School of Medicine, and the Clinical Microbiology Laboratory, Grady Memorial Hospital, Atlanta, Georgia.

Address reprint requests to John E. McGowan, Jr., MD, Director, Clinical Microbiology (Box 248), Grady Memorial Hospital, 80 Butler Street, Atlanta, GA 30335.
}

\section{How Good Are the Methods?}

In reviewing these trials, careful attention must be paid to the methods used for investigation. ${ }^{2}$ Certain problems stand out. First, laboratory techniques used to test for susceptibility differ from hospital to hospital and from time to time. For example, Gerding and Larson ${ }^{20}$ mention that data from the first several years of amikacin use are difficult to compare with current information because disks used earlier in disk agar-diffusion studies had a different concentration of amikacin than the disks used today. However, many of the studies listed in the Table compensated for laboratory variability by referring most resistant organisms to a central testing facility.

Second, selection biases are likely in studies of this subject. For example, much of the information about switching aminoglycosides comes from studies sponsored by a company making one of the drugs suggested for this indication. Most of the papers listed in the Table were from two symposia sponsored by the manufacturer. Can independent studies of the issue receive the same access to publication?

A third factor to consider is the control of confounding variables. Antimicrobial usage has often been assumed to be the sole factor responsible for changes in resistance patterns. However, a number of other factors can be involved as well. ${ }^{2,21}$ These factors include changes in the patient population (especially prominent and potentially dramatic in these days of "hospital marketing plans"), 
TABLE

CHANGES IN PREVALENCE OF RESISTANCE IN GRAM-NEGATIVE AEROBIC BACILLI ASSOCIATED

WITH CHANGES IN USE OF AMINOGLYCOSIDE ANTIMICROBIALS IN SEVERAL STUDIES, 1980-1986*

\begin{tabular}{|c|c|c|c|c|c|c|}
\hline \multirow[b]{2}{*}{ Author } & \multicolumn{3}{|c|}{ Change in use of } & \multicolumn{3}{|c|}{ Change in resistance to } \\
\hline & Gent $†$ & Tobr & Amik & Gent & Tobr & Amik \\
\hline Berk 5 & $D \ddagger$ & $D$ & 1 & $\mathrm{D}$ & $\mathrm{D}$ & $S$ \\
\hline Yurasek 6 & $D$ & & I & $\mathrm{D}$ & & S \\
\hline Moody ${ }^{7}$ & $\mathrm{D}$ & & I & $\mathrm{D}$ & & S \\
\hline Wielunsky 8 & $D$ & & 1 & $\mathrm{D}$ & & \\
\hline Cross $^{9}$ & 1 & $S$ & 1 & 1 & 1 & 1 \\
\hline Betts $^{10}$ & $\mathrm{D}$ & & 1 & $\mathrm{D}$ & $\mathrm{D}$ & $S$ \\
\hline \multicolumn{7}{|l|}{ Saravolatz ${ }^{11}$} \\
\hline Period 1 & $\mathrm{D}$ & & 1 & $D$ & & \\
\hline Period 2 & 1 & & $D$ & 1 & & \\
\hline Shulman 12 & $D$ & $D$ & । & $S$ & S & $S$ \\
\hline Levine $^{13}$ & S & 1 & I & $\mathrm{S}$ & $S$ & 1 \\
\hline \multicolumn{7}{|l|}{ Gerding 14} \\
\hline 14 VA Hosps & $\mathrm{D}$ & $\mathrm{D}$ & I & $\mathrm{D}$ & D & 1 \\
\hline Mpls VA Period 1 & $D$ & $D$ & I & $D$ & $D$ & $\mathrm{~S}$ \\
\hline Mpls VA Period 2 & I & & D & 1 & 1 & $\mathrm{~S}$ \\
\hline Mpls VA Period 3 & $D$ & & 1 & $D$ & $D$ & $D$ \\
\hline \multicolumn{7}{|l|}{ Young 15} \\
\hline Period 1 & $D$ & & 1 & $D$ & & $S$ \\
\hline Period 2 & 1 & & D & 1 & & 1 \\
\hline Acar $^{16}$ & & & 1 & & & V \\
\hline Saavedra ${ }^{17}$ & $D$ & & 1 & $\mathrm{D}$ & $D$ & 1 \\
\hline Ruiz-Palacios ${ }^{18}$ & D & $S$ & 1 & D & D & D \\
\hline Van Landuyt 19 & $D$ & $D$ & I & $\mathrm{D}$ & $\mathrm{D}$ & $\mathrm{D}$ \\
\hline
\end{tabular}

changes in the procedures and techniques performed (again, changing widely in response to the new economic forces), increased influence of organisms causing community-acquired infection (arising in part from today's rush to move the patient out of the hospital), and differences in prevalence of organisms from hospital to hospital. Such non-antibiotic factors may explain in part why increases in prevalence of resistant organisms are so strikingly location-specific. ${ }^{22}$

The studies listed in the Table attempted to detect differences in prevalence of resistance after changes in antimicrobial usage. Such investigations of temporal association require additional cautions. ${ }^{3}$ First, in similar studies, increase or decrease in resistance have occurred independent of changes in drug usage. ${ }^{23-26}$ Second, other control measures may be put into effect along with changes in antibiotic use. This makes it more difficult to attribute a causal role to altered antibiotic prescribing. ${ }^{3,22}$ Third, even when resistant organisms have been found to emerge, they do not necessarily persist. ${ }^{27}$ Fourth, even a marked increase in resistance at a given institution may increase the level only to levels seen at other institutions years ago. ${ }^{28}$ Fifth, a relationship may be apparent for some organisms but not for others. ${ }^{2,7,15,16,18,19,22,29}$ Sixth, it is difficult to analyze use of one drug without considering all other antimicrobials employed. As Mayer notes, "since plasmids may contain multiple resistance genes, each coding for an enzyme that inactivates one or more antibiotics, and since multiple drugs are used in the hospital environment, direct correlation of the use of specific antibiotics and specific resistance patterns may be obscured."22

\section{Can Switching Drugs Reduce Resistance?}

In several of the trials, a switch to amikacin from ge.. tamicin or tobramycin produced apparent decreases in prevalence of resistance to gentamicin or tobramycin without increase in amikacin resistance (see Table). Thus, hospitals experiencing outbreaks with gentamicin or tobramycin-resistant organisms well might try substituting amikacin in attempting to stem the epidemic.

In some trials, however, an increase in prevalence of amikacin resistance was seen during amikacin use, $9,13,14,16,17$ and an increase in prevalence of amikacin resistance has even been noted when the drug has not been employed. ${ }^{23}$ In countries where amikacin has been . introduced into routine use, the prevalence of resistance seems to have increased. ${ }^{30}$

When increased resistance has been noted, it has not necessarily occurred in a straight-line, dose-response relationship. Linear relationships have been noted on occasion, ${ }^{31}$ but the interval between introduction of an aminoglycoside into routine use and appearance of resistant strains in hospital populations has varied markedly for different drug-organism pairs. ${ }^{2,10}$ Occasionally, resistance has developed shortly after introduction of the 
drug; more frequently, a drug has been used for an appreciable period before resistance becomes prevalent. $16,29,32$

Plasmid-mediated resistance mechanisms have been demonstrated for amikacin ${ }^{33-35}$ as well as for other aminoglycosides. ${ }^{36,37}$ These observations help to explain the dramatic increases in resistance to amikacin that have occurred in "localized outbreaks," even after many years in which little or no increase in resistance was seen. ${ }^{16}$ Thus, whether or not switching or cycling of antimicrobials will be effective in reducing prevalence of resistance seems to depend on the organisms causing nosocomial infection in a given hospital and the types of resistance mechanisms present in these endemic bacteria.

\section{What About Cycling?}

Cycling is based on the hypothesis that substituting a new drug will foster decreased levels of resistance to the drugs formerly in use, restoring their value so that they again can be useful in therapy. The Table presents studies in which switching drugs did lead to a decrease in resistance to the older aminoglycosides. However, it is unclear that one can go back to the older drugs in the future. Data about this are scarce; in three studies that attempted to return to gentamicin from amikacin as the routine therapy, the prevalence of resistance to gentamicin rose again quickly after its use was reinstated.11,14,15

\section{Should I Cycle Antimicrobials in My Hospital?}

Whether or not to make the attempt to cycle aminoglycosides depends on several considerations ${ }^{3}$ :

First, does a problem of resistance exist? Resistant hospital organisms apparently exist to a much lesser extent in community hospitals. ${ }^{32,38,39}$ In view of the high cost of 'many of the alternative drugs, it makes no sense to introduce a new agent if no difficulty is being seen. ${ }^{40} \mathrm{By}$ contrast, prevalence of resistance is increasing in a number of university and other referral centers. ${ }^{41}$

Second, is the hospital's problem an organism or group of organisms known to have responded to switching or cycling at other institutions? As noted above, responses have been organism-specific. For some bacteria, substituting may not be as effective as limiting total prescriptions and duration of use for a given drug group-or for more than one group, if the resistance is plasmid-mediated and linked to other resistance factors. ${ }^{42}$

Third, what is the goal of the substitution? A goal of decreasing the selective pressure favoring an epidemic hospital organism seems potentially achievable, as noted frequently in the Table. However, the data presented above pose questions about the value of introducing one drug in an attempt to restore the utility of a second drug.

\section{Are These Practices Cost-Effective?}

A new emphasis on cost of antimicrobials has been spurred by prospective reimbursement. ${ }^{43}$ True costeffective use must consist of reducing antimicrobial use where the quality of care is not compromised. This stipulation must not be overlooked in the current efforts to diminish the overall level of prescribing of antimicrobials, which represent a large proportion of the pharmacy budget in many United States hospitals. To heed this rule, however, demands that we define cost-control in the context of improving antimicrobial use, emphasizing the areas of improvement which allow reduction of use.

That such improvement is possible is emphasized by Aldis and Cowan. ${ }^{43}$ These investigators studied the use of antimicrobials in a community hospital during a 1-month period, and found that changes in the antimicrobials employed could have been made without diminishing care of the patient, but with a substantial savings in cost to the hospital.

The studies to date provide minimal data on the impact of substitution or cycling programs on patient care, pharmacy acquisition and preparation costs, or drug administration costs. Thus, no conclusion about cost-effectiveness is possible at this time.

\section{CONCLUSIONS}

1. Antimicrobial usage is one, but only one, of the factors that leads to antimicrobial resistance in microorganisms.

2 . The relative potential of an antimicrobial for triggering resistance will help determine the drug's value ${ }^{3}$ - but efficient methods for this assessment are sadly lacking at present. ${ }^{41}$ Development of such methods should be a high research priority. 45

3. Decreasing overall antimicrobial use has been given special importance in today's prospective reimbursement system. ${ }^{46}$ These efforts also can help to minimize the likelihood of antimicrobial resistance. Antimicrobial usage must be reduced where quality of care will not be compromised. Yet, the phenomenon of linkage of resistance factors makes it uncertain that decreased use will lead to a drop in prevalence of resistance to that drug. ${ }^{13,47}$

4. The value of rotation or cycling of antimicrobials will depend on local patterns of resistant organisms, prevalence of specific resistance mechanisms in a given population of nosocomial organisms, and the goals set for such change in prescribing practices.

5. The cost-effectiveness of cycling programs is not evaluable at this time.

\section{REFERENCES}

1. McGowan JE Jr: Changing etiology of nosocomial bacteremia and fungemia and other hospital-acquired infections. Rev Infect Dis 1985; 7(Suppl 3):S357S370.

2. McGowan JE Jr: Antimicrobial resistance in hospital organisms and its relation to antibiotic use. Rev Infect Dis 1983; $5: 1033-1048$.

3. McGowan JE Jr: Is antimicrobial resistance in hospital microorganisms related to antibiotic use? Bull NY Acad Med (in press).

4. Levin S, Karakusis PH: Future trends in aminoglycoside therapy. Am J Med $1986 ; 80$ (Suppl 6B):190-194.

5. Berk SL, Alvarez S, Ortega $G$, et al: Clinical and microbiologic consequences of amikacin use during a 42-month period. Arch Intern Med 1986; 146:538-541.

6. Yurasek MC, Wang WL, Mostow SR: Reduction in gentamicin resistance among gram-negative bacilli with the exclusive use of amikacin. Clin Res 1981; 29:92A (abstract)

7. Moody MM, de Jongh CA, Schimpff SC, et al: Long term amikacin use: Effects on aminoglycoside susceptibility patterns of gram-negative bacilli. JAMA 1982; 248:1199-1202.

8. Wielunsky E, Drucker M, Cohen T, et al: Replacement of gentamicin by amikacin as a means of decreasing gentamicin resistance of gram-negative rods in a neonatal intensive care unit. Isr J Med Sci 1983; 19:1006-1008.

9. Cross AS, Opal S, Kopecko DJ: Progressive increase in antibiotic resistance of gram-negative bacterial isolates: Walter Reed Hospital, 1976 to 1980: Specific analysis of gentamicin, tobramycin, and amikacin resistance. Arch Intern Med $1983 ; 143: 2075-2080$

10. Betts RF, Valenti WM, Chapman SW, et al: Five-year surveillance of aminogly- 
coside usage in a university hospital. Ann Intern Med 1984; 100:219-222.

11. Saravolatz LD, Arking L, Pohlod D, et al: An outbreak of gentamicin-resistant Klebsiella pneumoniae: Analysis of control measures. Infect Control 1984; 5:79-84.

12. Shulman ST, Yogev R: Treatment of pediatric infections with amikacin as firstline aminoglycoside. Am J Med 1985; 79(Suppl 1A):43-50.

13. Levine JF, Maslow M], Leibowitz RE, et al: Amikacin-resistant gram-negative bacilli: Correlation of occurrence with amikacin use. I Infect Dis 1985; 151:295-300.

14. Gerding DN, Larson TA: Aminoglycoside resistance in gram-negative, bacilli during increased amikacin use. Am J Med 1985; 79(Suppl 1A):1-7.

15. Young E], Sewell CM, Koza MA, et al: Antibiotic resistance patterns during aminoglycoside restriction. Am J Med Sci 1985; 290:223-227.

16. Acar JF, Goldstein FW, Menard R, et al: Strategies in aminoglycoside use and impact on resistance. Am J Med 1986; 80(Suppl 6B):82-87.

17. Saavedra S, Vera D, Ramirez-Ronda CH: Susceptibility of aerobic gram-negative bacilli to aminoglycosides-Effects of 45 months of amikacin as first-line aminoglycoside therapy. Am J Med 1986; 80(Suppl 6B):65-70.

18. Ruiz-Palacios GM, Ponce de Leon S, Sifuentes J, et al: Control of emergence of multi-resistant gram-negative bacilli by exclusive use of amikacin. Am J Med 1986; 80(Suppl 6B):71-75.

19. Van Landuyt HW, Boelaert J, Glibert B, et al: Surveillance of aminoglycoside resistance-European data. Am J Med 1986; 80(Suppl 6B):76-81.

20. Gerding DN, Larson TA: Resistance surveillance programs and the incidence of gram-negative bacillary resistance to amikacin from 1967 to 1985 . Am J Med $1986 ; 80$ (Suppl 6B):22-28.

21. Young LS, Hindler J: Aminoglycoside resistance: A worldwide perspective. $A m J$ Med 1986; 80(Suppl 6B):15-21.

22. Mayer KH: Review of epidemic aminoglycoside resistance worldwide. Am J Med 1986; 80(Suppl 6B):56-64.

23. Bryan CS, Parker E, Schoenlein PV, et al: Plasmid-mediated antibiotic resistance in a changing hospital environment: Efficacy of control measures. Am J Infect Control 1980; 8:65-71.

24. John JF Jr, Rubens CE, Farrar WE Jr: Characteristics of gentamicin resistance in nosocomial infections. Am J Med Sci 1980; 279:25-30.

25. Weinstein RA, Nathan C, Gruensfelder R, et al: Endemic aminoglycoside resistance in gram-negative bacilli: Epidemiology and mechanism. J Infect Dis $1980 ; 141: 338-345$

26. Lane MM, Parker DE, Flournoy DJ: Trends in bacterial susceptibility to antimicrobial agents. Chemotherapy 1985; 31:433-439.

27. Mickelsen PA, Plorde JJ, Gordon KP, et al: Instability of antibiotic resistance in a strain of Staphylococcus epidermidis isolated from an outbreak of prosthetic valve endocarditis. I Infect Dis 1985; 152:50-58.

28. Cherubin CE, Eng RHK, Dabernat H, Lareng MB: Comparison of antibiotic resistance of gram-negative bacilli with beta-lactam and aminoglycoside antibiotics. Diagn Microbiol Infect Dis 1986; 4:153-159.

29. Daschner $\vec{F}$, et al: Antibiotic resistance in intensive care unit areas. Infect Control 1983: 4:382-387.
30. Shimizu K, Kumada T, Hsieh W, et al: Comparison of aminoglycoside resistance patterns in Japan, Formosa, and Korea, Chile, and the United States. Antimicrob Agents Chemother 1985; 28:282-288.

31. Goossens H, Van Laethem Y, Levy J, et al: Predicting gentamicin resistance from annual usage in hospital. Lancet 1986; 2:804-805.

32. Magnussen CR, Jacobson MT: Longitudinal analysis of endemic gentamicin and tobramycin-resistant gram-negative bacilli in a community hospital. Infect Control 1984; 5:88-92.

33. Van Nhieu GT, Goldstein FW, Pinto ME, et al: Transfer of amikacin resistance by closely related plasmids in members of the family Enierobacteriaceae isolated in Chile. Antimicrob Agents Chemother 1986; 29:833-837.

34. Tolmasky ME, Roberts $M$, Woloj $M$, et al: Molecular cloning of amikacin resistance determinants from a Klebsiella pneumoniae plasmid. Antimicrob Agents Chemother 1986; 30:315-320.

35. Phillips I, King A, Shannon K: Prevalence and mechanisms of aminoglycoside resistance. A ten-year study. Am J Med 1986; 80(Suppl 6B):48-55.

36. Lee SC, Gerding DN, Cleary PP: Hospital distribution, persistence, and reintroduction of related gentamicin $\mathrm{R}$ plasmids. Antimicrob Agents Chemother $1986 ; 29: 654-659$.

37. Larson TA, Garrett CR, Gerding DN: Frequency of aminoglycoside 6'-Nacetyltransferase among Serratia species during increased use of amikacin in the hospital. Antimicrob Agents Chemother 1986; 30:176-178.

38. Isenberg HD, Berkman JI: The role of drug-resistant and drug-selected bacteria in nosocomial disease. Ann NY Acad Sci 1971; 182:52-58.

39. Gardner DA, Lorian V: Bacterial susceptibility to antibiotics remains virtually stable. Antimicrobic Newsletter 1985; 2:9-13.

40. Manu P: Empiric use of aminoglycosides in hospitals. Ann Intern Med 1984; 100:919.

41. Sanders CC, Sanders WE Jr: Microbial resistance to newer generation betalactam antibiotics: Clinical and laboratory implications. I Infect Dis 1985; 151:399-406.

42. Woloj M, Tolmasky ME, Roberts MC, et al: Plasmid-encoded amikacin resistance in multiresistant strains of Klebsiella pneumoniae isolated from neonates with meningitis. Antimicrob Agents Chemother 1986; 29:315-319.

43. Neu HC: Antimicrobial activity, bacterial resistance, and antimicrobial pharmacology: Is it possible to use new agents cost-effectively? Am J Med 1985; 78(Suppl 6B):17-22.

44. Aldis WL, Cowan D: Cost-effectiveness of antibiotic use. JAMA 1984; $252: 3252$.

45. Jorgensen JH: Susceptibility test methods which may be able to predict the emergence of resistance to newer beta-lactam antibiotics. Chemioterapia 1985 ; 4:7-13.

46. Quintiliani R, Klimek JJ, Nightingale CH: Restriction policies for therapy with combination antibiotics. J Infect Dis 1986; 153:645-647.

47. Schaberg DR, Power G, Betzold J, et al: Conjugative $R$ plasmids in antimicrobial resistance of Staphylococcus aureus causing nosocomial infections. Infect Dis 1985; 152:43-49. 